\title{
Allowing Errors in Speech over Wireless LANs
}

\author{
Ian Chakeres, Hui Dong, Elizabeth Belding-Royer ${ }^{\ddagger}$, Allen Gersho, Jerry Gibson \\ University of California, Santa Barbara \\ Dept. of Electrical and Computer Engineering \\ Dept. of Computer Science $\ddagger$ \\ idc@engineering.ucsb.edu, \{huidong, gersho, gibson\}ece.ucsb.edu, ebelding@cs.ucsb.edu
}

\begin{abstract}
Wireless LANs are becoming commonplace because of their ease in providing mobile communication. As use of IEEE 802.11 continues to expand, support for voice will become a desirable feature. Supporting voice conversations over a packet network is more challenging than over current circuit switched wired networks. These challenges are particularly difficult due to the erroneous nature of a wireless channel. When errors occur in data packets the IEEE 802.11 MAC layer standard requires that the receiver not accept the packet; the source must retransmit all packets received with errors. However, digitized voice data can tolerate some error and loss without noticeable degradation in call quality. By forcing error-free reception of speech, scarce bandwidth and energy are unnecessarily expended, and the delay of the packets increases. In this paper we explore allowing bit errors in voice packets over IEEE 802.11 networks. We propose two strategies for allowing these errors and demonstrate that they both result in improved performance.
\end{abstract}

\section{INTRODUCTION}

Wireless LANs (WLAN) based on the IEEE 802.11 standard are experiencing widespread deployment. Currently most devices with WLAN connectivity are laptops or handheld devices; however, there is a new generation of IEEE 802.11 phone devices reaching the market. These devices promise voice conversations over IEEE 802.11 networks, thereby unifying the voice and data networks while allowing mobility.

Sending voice over an Internet Protocol packet network (VoIP) is considerably different from the circuit-switched networks that are typically used for voice conversations. In a circuit-switched network, bandwidth is dedicated and delay is minimal. In IP packet networks there are no guarantees; all packets must compete and share the same bandwidth, resulting in packet loss, delay and jitter.

Voice call quality degrades as packet loss, delay and jitter increase. Packet loss influences the quality of the received voice conversation; extreme losses may render the speech unintelligible. Likewise, as delay increases the interaction between call participants becomes more difficult. Jitter, the variance in the inter-arrival time between packets, must be kept small; otherwise, buffering must be used, which increases the overall delay. In order to maintain normal voice conversations, the loss, delay and jitter must therefore be kept within tight bounds.

Wireless channels characteristically have high error rates, particularly when compared with wired networks. These errors are due to many factors, including time varying properties, obstacles, distance, collisions, and multi-path interference. The IEEE 802.11 MAC layer standard does not allow (or correct) any errors in packet transmissions. If any error occurs during the hop-wise transmission of a unicast packet, the sender is required to retransmit the packet. A maximum of seven attempts is allowed for a single packet.

We argue that when transmitting voice packets, errors within these packets should be permissible. Because voice can tolerate packet loss and error ${ }^{1}$, allowing some bit errors does not impact the perceived conversation quality. Allowing bit errors in voice packets has the benefit of reducing the number of retransmissions per packet. This, in turn, reduces the delay and jitter. In congested networks, fewer retransmissions reduce the channel usage and result in increased packet delivery.

In this paper we describe a mechanism for allowing bit errors in voice packets in IEEE 802.11 networks. We perform simulation experiments with VoIP conversations to determine the impact of allowing errors at the medium access control (MAC) layer in the voice data. We show that in a lossy environment allowing errors improves the performance and in some cases allows a higher number of calls to take place concurrently. Specifically, the contributions of this paper are the following:

- Proposal of two IEEE 802.11 MAC layer designs allowing bit errors

- Performance evaluation measuring performance gains

- Thorough examination of many common traffic and channel conditions using the proposed MAC layer designs

The outline for the remainder of the paper is as follows. Section II describes related work. It is followed by an overview of VoIP, IEEE 802.11 and the error model in Section III. Section IV describes the modifications to the system to allow bit errors. The experimental setup is described in Section V. The results from simulation are discussed in Section VI. Finally Section VII concludes the paper.

\footnotetext{
${ }^{1}$ To conceal packet loss and error, various techniques may be employed, such as error correction or multirate coding. Many techniques to hide packet loss and error for VoIP applications are provided by [1].
} 


\section{RELATED WORK}

The support of VoIP conversations in WLANs is very challenging. VoIP has unique requirements that were not considered when IP packet networks were developed. In the system considered here, modifications to improve IEEE 802.11 and VoIP result in higher performance and a better user experience.

Improvements in voice encoders such as lower bit rates [2], error correction, and error and loss concealment [3], [4] are most likely to impact the ability to support multiple concurrent calls with high quality. Likewise, improvements to IEEE 802.11 such as higher data rates [5], QoS [6], improved channel use and fairness increase the performance of the overall system. In general, for voice delivery, these improvements can be combined with a MAC layer that allows bit errors to further increase performance.

There has been significant research exploring the effect of packet losses due to wireless channel errors on TCP [7], [8], [9]. Most of this research has focused on how to hide errors by modifying the link layer behavior, such as link layer retransmissions and adaptive packet sizes. This research has not examined the effect of bit errors because TCP is a reliable protocol; no errors are tolerated.

In [10] the authors examine methods to protect important packets. They require the important packets to be transmitted at IEEE 802.11 b's lowest bit rate because packets transmitted at this lowest bit rate have the largest range and are more likely to be received without errors. In addition the authors perform redundant packet transmissions to increase the likelihood of reception. These techniques may be used in combination with the MAC layer CRC schemes we propose to further improve performance.

In [11] the authors introduce UDP Lite, a transport layer that allows partial checksumming of the UDP data packet contents. In UDP Lite, the UDP header is modified to indicate which portion of the packet is protected by the UDP checksum. This allows bit errors in part of the packet while important application information is still protected. This scheme is similar to our proposal, with the exception that UDP Lite is performed at the transport layer. Therefore, when UDP Lite is used over WLANs, no errors would occur and no improvement would be seen, since the IEEE 802.11 MAC layer does not pass packets with errors up the protocol stack. To experience improvement when UDP Lite is used over WLANs, modifications, as proposed in this paper, would need to be implemented. The UDP lite authors did not examine the impact of bit errors in erroneous wireless networks, nor did they experiment with a modified MAC layer.

\section{SySTEM MODEL}

Before describing the design details we highlight the key components that must be considered when discussing VoIP in
TABLE I

VOICE ENCODER BIT RATES.

\begin{tabular}{|c|c|}
\hline Encoder & Bit Rate \\
\hline \hline G.711 & 64 kbps \\
\hline G.723.1 & $5.3,6.3 \mathrm{kbps}$ \\
\hline
\end{tabular}

IEEE 802.11 networks. First, we describe VoIP and the speech encoders we utilized in our experiments. Next, we describe the MAC layer operation of the IEEE 802.11 standard. Finally we discuss the Gilbert error model, which is used to simulate bit errors in packets that are transmitted over the wireless medium.

There are two main components to perform a VoIP call: call signaling and voice data delivery. In this paper we focus solely on data delivery. We do not consider call signaling (setup, transfer, tear down, etc.). More information on this topic can be found in [12], [13].

\section{A. Voice Encoders}

To transmit voice over a packet network, the analog speech must first be encoded and packetized. The voice encoder determines the bit rate of the encoded voice. The voice encoder/decoder pair also influences the robustness of the voice conversation in the presence of errors and losses [14].

There are numerous encoders for speech. One of the most widely used voice encoders is G.711 [15], as it was an early standard. G.723.1 [2] is an advanced speech coder that offers a much lower bit rate. The bit rates for these two encoders are shown in Table I.

G.711 digitizes speech using pulse code modulation (PCM). Speech is sampled at $8 \mathrm{khz}$ and each sample is 8 bits. This leads to a bit rate of $64 \mathrm{kbps}$, or 8 bytes per millisecond (ms). Because the G.711 encoder creates a bit stream, the voice stream can be framed at any size. Typically the G.711 bit stream is framed into packets containing $20 \mathrm{~ms}$ of speech. This value is chosen because the frame size of the packet influences the overall delay. With a $20 \mathrm{~ms}$ frame size, each data packet contains 160 bytes. G.711 is also capable of silence suppression, where data is not sent when no speech is detected.

G.723.1 has two bit rates. For the $6.3 \mathrm{kbps}$ encoding rate, a multi-pulse-maximum likelihood quantization (MPMLQ) is performed to encode the speech. At the lower data rate algebraic-code-excited linear prediction (ACELP) is employed. G.723.1 is also capable of silence suppression. Each data frame contains $30 \mathrm{~ms}$ of voice and a small $7.5 \mathrm{~ms}$ lookahead buffer is required.

\section{B. IEEE 802.11 Standard}

The IEEE 802.11 standard [16] is widely used in WLAN deployments. In this standard, when a node has a packet to send, it first performs carrier sensing (CS). If the medium is idle for a small period of time, then the packet is transmitted. 


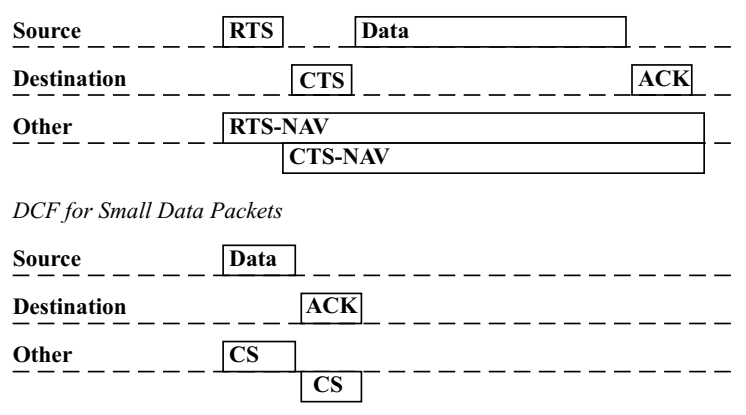

Fig. 1. IEEE 802.11 distributed coordination function.

If the medium is busy, the node must backoff. A random backoff time, called the contention window, is chosen. This contention window is only decremented while the medium is idle. When the backoff timer expires the node transmits the packet. If the node does not receive acknowledgment that the packet was received correctly, then the node must backoff again and attempt retransmission. Similar to Ethernet, an exponential backoff is utilized. After seven unsuccessful attempts, an unsent packet is dropped.

When transmitting unicast data packets, the IEEE 802.11 MAC layer utilizes the distributed coordination function (DCF). DCF consists of a RTS-CTS-Data-ACK handshake, as shown in Figure 1. The Request to Send (RTS) and Clear to Send (CTS) packets are small and therefore decrease the impact of collisions. These packets are used to perform virtual carrier sensing to avoid the hidden terminal problem [17]. Virtual carrier sensing causes nodes that overhear the RTS or CTS packets to defer their packet transmissions during the period of transmission.

When the data packet itself is small, RTS and CTS packets are not used because they increase overhead and actually hinder performance [18]. This is the case for voice data. When RTS and CTS packets are not used, other nodes avoid transmission because, prior to sending, they perform carrier sensing. If they sense the medium is in use they will not decrease their contention window and therefore not send any packets at that time. Figure 1 shows carrier sensing as it is performed by nodes overhearing the Data or ACK packets. The ACK packet is still utilized to confirm the packet was received without error. If there is any error detected in the packet, the destination does not send an ACK, and the source is required to retransmit the packet.

\section{Gilbert Error Model}

The Gilbert error model [19] utilizes a first order Markov model for data transmission on fading channels. This model has been shown to be a good approximation of the errors that occur on a wireless channel [20]. The Gilbert error model is a bursty error model. This results in periods of time where there are bursts of errors. Likewise there are periods of time where there are no errors.

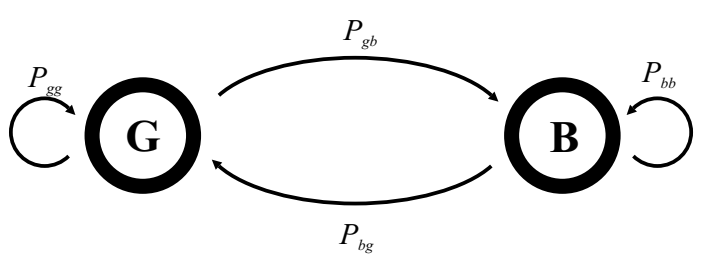

Fig. 2. Gilbert error model state diagram.

The Gilbert error model state diagram is shown in Figure 2. There are two states, G (for good) and B (for bad or burst). When in the state G, $P_{g g}$ is the probability of staying in the state $\mathrm{G}$, while $P_{g b}$ is the probability of transitioning from state G to B. Hence, $P_{g b}=1-P_{g g}$. The error model stays in the current state for $\log \left(r_{l}\right) / \log (P)$ bits, where $r_{l}$ is a random number between zero and one, and $P$ is the probability of staying in the current state. After $\log \left(r_{l}\right) / \log (P)$ bits have been transmitted a state change occurs if $r_{s}$ is greater than $P_{g g}\left(P_{b b}\right)$ and the error model is currently in state G (B), where $r_{s}$ is a random number between zero and one. If $r_{s}$ is less than $P_{g g}\left(P_{b b}\right)$ no state change occurs. Either way, the error model will stay in the chosen state for $\log \left(r_{l}\right) / \log (P)$ bits. There are two other parameters not represented on the figure, $P_{e g}$ and $P_{e b}$. $P_{e g}\left(P_{e b}\right)$ is the probability that a bit is in error while in state $G(B)$.

Given these values the average bit error rate (ABER) can be calculated by the following equation [21]:

$$
\frac{P_{e g}\left(1-P_{g g}\right)}{\left(1-P_{g g}\right)+\left(1-P_{b b}\right)}+\frac{P_{e b}\left(1-P_{b b}\right)}{\left(1-P_{g g}\right)+\left(1-P_{b b}\right)}
$$

Though the ABER gives an estimate of the BER and can be used to compare the average performance of different models, it does not correctly describe the error behavior because the Gilbert error model is highly bursty. The errors occur in bursts when the model is in state B. Hence, the ABER does not accurately describe expected losses. For example, given a ABER of $1 \%$, one would not expect 1 bit error in every 100 bits. Rather, one might expect no bit errors in state $\mathrm{G}$ and a cluster of bit errors when state B.

In order to utilize the Gilbert error model, values for following parameters must be chosen: $P_{g g}, P_{b b}, P_{e g}$ and $P_{e b}$. In [22], experiments were performed to determine estimate values for the Gilbert error model parameters. The authors examined four cases:

- Average

- Noise Limited (NL)

- Interference Limited (IL)

- Frequency Selective Fading Limited (FSFL)

Table II shows the determined parameter values and ABER. FSFL results in the fewest bit errors, while IL results in the most bit errors. We expect the average and NL cases to exhibit similar error characteristics because they have the same ABER, though in slightly different burst lengths. 
TABLE II

Gilbert ERror MOdel PARAMETERs.

\begin{tabular}{|c|c|c|c|c|c|}
\hline Model & $P_{g g}$ & $P_{e g}$ & $P_{b b}$ & $P_{e b}$ & $A B E R$ \\
\hline \hline Average & $99 \%$ & $0 \%$ & $85 \%$ & $33 \%$ & $1 \%$ \\
\hline NL & $97 \%$ & $0 \%$ & $75 \%$ & $28 \%$ & $1 \%$ \\
\hline IL & $99 \%$ & $0 \%$ & $95 \%$ & $42 \%$ & $8 \%$ \\
\hline FSFL & $99 \%$ & $0 \%$ & $75 \%$ & $38 \%$ & $0.3 \%$ \\
\hline
\end{tabular}

\section{Design to Allow Bit Errors}

The main influences on perceived voice quality are packet loss, delay and jitter. Voice requirements are significantly different from many other applications; comparing voice and file transfer requirements, file transfer is delay and jitter tolerant. Additionally, file transfer data cannot contain any losses or errors. On the other hand, voice packets can tolerate some loss and error by utilizing loss concealment techniques. Delay and jitter must be small since they significantly impact interactive communication.

Because the IEEE 802.11 MAC layer protocol was designed to handle applications such as file transfer, it requires that there are no errors in received packets. For voice, this requirement is unnecessary because voice can tolerate errors and losses. Therefore, we propose a modification to the IEEE 802.11 MAC layer to allow errors in the data portion of received voice packets.

In this section we describe the normal operation of $C R C \mathrm{~s}$ and checksums utilized to guarantee the accuracy of the packet. Next, we present our modification to the IEEE 802.11 MAC layer to allow bit errors. Additionally, we propose a MAC layer optimization to minimize packet loss while still allowing bit errors in the voice data. Finally we describe how these modifications might be utilized in a real implementation.

\section{A. CRCs and Checksums}

Cyclic redundancy checks (CRC) and checksums are used at many layers of the network stack to ensure the contents of certain parts of the packet are correctly received and free of errors. For instance, the IEEE 802.11 MAC protocol, Internet Protocol (IP) and User Datagram Protocol (UDP) all contain CRCs or checksums that verify part of the packet.

To ensure error-free reception of packets, the IEEE 802.11 MAC layer has a CRC that is calculated over the entire packet, including the IP and UDP headers and voice data, as shown in Figure 3. If there is any error in a packet when it is received

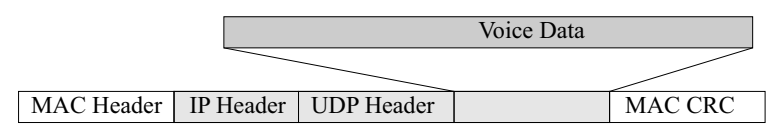

Fig. 3. MAC layer frame contents. at the MAC layer, it fails the CRC and it is discarded. This results in a MAC layer retransmission.

At the network layer, the Internet Protocol (IP) header contains a checksum that protects only the IP header. If, upon reception at each hop, this checksum does not match the value contained in the IP header, an error has occurred and the packet must be dropped. If the packet was not dropped, the data might be routed to an incorrect destination. The IP header checksum does not protect the UDP header or data from errors.

At the transport layer, the UDP header contains a checksum protecting the UDP header, an IP pseudo-header and data. This checksum may be disabled by placing zero in the checksum field. This can be accomplished by using a RAW socket or a specialized UDP implementation [11]. However, the UDP checksum is typically computed. This ensures that the UDP header, IP pseudo-header and data are received without error. Errors in the UDP header could result in packets being passed to the wrong application port, resulting in packet losses.

We refer to the typical operation of these layers as T-CRC. In T-CRC, the MAC layer computes a CRC over the entire packet. The IP protocol computes a checksum over the IP header and the UDP checksum protects the UDP header, IP pseudo-header and data.

\section{B. MAC Layer Header CRC}

To improve voice performance in IEEE 802.11 networks, we propose to allow errors in the data portion of speech packets. To accomplish this, the MAC layer CRC must not protect the data portion of the packet; however, the MAC header should still be protected. Otherwise, if the MAC header contains errors, packets may be received by an incorrect destination. By protecting only the MAC header with a CRC, the MAC layer header is guaranteed to be correct, but the contents of the data may contain errors. After this minor change to IEEE 802.11, if there are errors in the data of the MAC layer packet, no retransmission occurs. However, if a packet is received with an error in the MAC header, the packet is dropped and a retransmission occurs. We refer to this strategy as M-CRC.

In addition to modifying the MAC layer CRC, the UDP checksum must be disabled to allow errors in voice packet data. Otherwise the UDP layer will discard packets containing errors. As previously stated, this can be accomplished by placing zero in the checksum field.

Note that in this strategy, because the IP and UDP headers are not protected by the MAC layer CRC, some packets will be lost. If there is an error in the IP header, the IP checksum will fail and the packet must be discarded; otherwise, the packet could be routed to the wrong destination. If there is an error in the UDP header then the packet will not be sent to the correct application port and will be considered lost. To combat these disadvantages we propose another MAC layer CRC calculation strategy, described in the following section. 


\section{Entire Header CRC}

For proper operation the MAC layer should guarantee the correctness of all control headers (and other data) that cannot tolerate errors. In our case this includes the MAC, IP and UDP headers. If additional bits within the UDP data packet require correct reception, these bits should also be protected by the MAC layer CRC. For example, when utilizing the Real-time Transport Protocol (RTP) and the Adaptive Multirate Coder (AMR) [3], the RTP header and AMR class 1a bits ${ }^{2}$ must be received without error. Therefore the MAC layer CRC should protect these bits in addition to the MAC, IP and UDP headers. Otherwise, packets with errors in these headers will be dropped at the upper layers, resulting in lower packet delivery. For example, if a packet is received with errors in the IP header, the MAC layer will accept the packet because there are no errors in the MAC header. However, the packet will then be dropped by the IP layer. There will not be a retransmission of the packet because according to the MAC layer, the packet has been successfully received.

If the MAC CRC protects all the headers (and other bits that cannot tolerate errors), then any corruption in these bits will cause immediate retransmission of the packet at the MAC layer. The result is a decrease in the number of packets lost, though additional MAC layer transmissions may be necessary. We refer to this strategy as E-CRC.

As in the case of M-CRC, in addition to allowing errors at the MAC layer, the UDP checksum must be turned off. Otherwise errors in the data portion of the UDP packet will not be allowed.

\section{MAC Layer Implementation}

To allow bit errors in the data portion of packets at the MAC layer, IEEE 802.11 must be modified. In networks where all traffic is voice data with the same protection requirements, the portion of the packet protected by the CRC could be fixed. Therefore the CRC behavior could be changed to protect a static portion of all packets. In this case, because the number of bits protected by the MAC layer CRC is fixed and all nodes calculate the CRC identically, it is not necessary to modify the IEEE 802.11 packet format.

Networks that support multiple data types require multiple MAC layer CRC protection strategies (such as T-CRC, M$\mathrm{CRC}$ and E-CRC). In these networks, other mechanisms are necessary to identify the portion of the packet that is protected by the MAC layer CRC. In the MAC or PLCP layer header, control bits can be introduced to indicate the portion of the packet protected by the CRC. When a packet is received, these bits are examined. Any error in the portion of the packet protected by the CRC will result in a CRC check failure and require a MAC layer retransmission. The main disadvantage

\footnotetext{
${ }^{2}$ AMR has multiple classes of bits. Bits in class 1a are needed for reconstruction and must be error free, while other bits can tolerate errors.
}

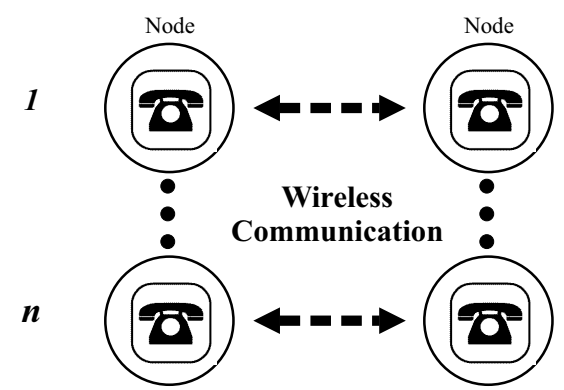

Fig. 4. Network and call scenario diagram. Each node has one VoIP client that sends and receives voice traffi $\mathrm{c}$ to one other node. In the network, there are $n$ bi-directional calls and $2 * n$ nodes in the network.

of this modification scheme is these modified control headers are not backward compatible with other IEEE 802.11 devices.

\section{EXPERIMENTS}

In this section, we describe the experimental settings for the various components in the system. We discuss the network and call topology, encoder and data framing, MAC level error checking, channel error models and other simulator parameters.

\section{A. Network and Call Topology}

In our simulations, we model a wireless LAN. Hence, all nodes are within range of each other and share the wireless medium. The network and call topology is shown in Figure 4. In this scenario, each node has a full duplex voice conversation with one other node. There are $2 * N$ nodes and $N$ full duplex calls.

TABLE III

Voice TrafFic Simulated.

\begin{tabular}{|c|c|c|c|}
\hline Encoding & $\begin{array}{c}\text { Bit } \\
\text { Rate }\end{array}$ & $\begin{array}{c}\text { Voice } \\
\text { per Packet }\end{array}$ & $\begin{array}{c}\text { Bytes } \\
\text { per Packet }\end{array}$ \\
\hline \hline G.723.1 & $6.3 \mathrm{kbps}$ & $30 \mathrm{~ms}$ & $24^{3}$ \\
\hline G.711 & $64 \mathrm{kbps}$ & $20 \mathrm{~ms}$ & 160 \\
\hline G.711 & $64 \mathrm{kbps}$ & $25 \mathrm{~ms}$ & 200 \\
\hline G.711 & $64 \mathrm{kbps}$ & $30 \mathrm{~ms}$ & 240 \\
\hline G.711 & $64 \mathrm{kbps}$ & $35 \mathrm{~ms}$ & 280 \\
\hline G.711 & $64 \mathrm{kbps}$ & $40 \mathrm{~ms}$ & 320 \\
\hline
\end{tabular}

\section{B. Voice Encoder and Data Framing}

We explore two data encoding rates: 64 and $6.3 \mathrm{kbps}$. These data rates are representative of G.711 and G.723.1, as described in Section III-A. The default voice packet frame sizes, $20 \mathrm{~ms}$ (160 bytes) for G.711 and $30 \mathrm{~ms}$ ( 24 bytes) for G.723.1, respectively, are utilized. In addition to examining

${ }^{3}$ G.723.1 packet frames contain 189 bits (23.625 bytes). An additional 3 bits were added to each frame to have byte alignment. This results in 24 byte packets. With this packet size the actual data rate is $6.4 \mathrm{kbps}$. 
the default values for these two encoders, we explore various packet frame sizes. The frame size determines the amount of speech data contained in each packet. Increasing the voice data in a packet while maintaining a constant bit rate results in fewer packets per second. The encoding rates and packet frame sizes we examine are shown in Table III.

\section{MAC Layer CRC}

Three strategies for the MAC layer CRC are explored:

- Typical IEEE 802.11 CRC (T-CRC): This is the IEEE 802.11 MAC layer without modification. The CRC is computed over the whole packet, including the data portion. Any error in the packet causes a MAC layer retransmission to occur.

- MAC Header CRC (M-CRC): In this strategy the MAC layer only computes a CRC over the IEEE 802.11 MAC header. Packets with errors in the MAC header are dropped and a MAC layer retransmission occurs.

- Entire Header CRC (E-CRC): This MAC layer CRC strategy computes a CRC over the MAC, IP and UDP headers. Packets with errors in any header are dropped and a MAC layer retransmission occurs.

These three strategies operate as described in Section IV.

\section{Error Models}

Because the CRC is only beneficial in a lossy environment, we utilize the Gilbert error model to introduce bit errors in our simulations. We simulate four different sets (Average, NL, IL, FSFL) of values for the Gilbert error model parameters, as was shown in Table II. These values were experimentally determined and represent realistic channel conditions [20].

\section{E. Other Simulation Settings}

Simulations were performed using the NS-2 simulator [23]. The data rate of IEEE 802.11 was configured to be 2 Mbps. The link layer queue was drop-tail with a capacity of 50 packets. Since all nodes were within range of each other, no routing protocol was necessary.

The voice conversations were simulated utilizing a constant bit rate traffic source. Because each call is bidirectional, there is one stream of voice packets flowing in each direction.

\section{F. Simulation Environment Summary}

We perform simulations to examine the effect of allowing bit errors on VoIP traffic. We vary the following parameters:

- MAC Layer CRC Strategy

- Number of Calls

- Voice Encoder

- Voice Frame Size

- Gilbert Error Model Parameters

Ten runs of each simulation were performed and averaged results are presented.
Unless otherwise stated the default simulation settings are: G.711 encoder, $20 \mathrm{~ms}$ voice frame, and the Average Gilbert error model parameters.

\section{RESUlTS AND DISCUSSION}

In this section, we discuss the results of the simulations performed. First we present our metrics for evaluation. Next, we describe the general trends for the three different MAC layer CRC strategies. Afterward, we show the effect of varying the voice encoder, voice frame size and channel error model parameters.

\section{A. Performance Metrics}

To evaluate the effect of allowing bit errors in voice packets we use the following metrics:

- Packet Delivery Ratio: This is the number of voice packets received divided by the number of packets sent. As the packet delivery ratio drops, the number of losses increases. As the number of losses increases, the quality of the voice conversation degrades.

- Delay: The one-way delay is the time between the transmission of the voice packet at the source and its reception at the destination. As the delay increases, the interactive nature of the conversation degrades. In practice there may be additional delay for data framing and queuing at the destination for playback; these are often static and not considered. Realistic bounds for delay are shown in Table IV.

- MAC Layer Transmissions per Packet Delivered: Ideally each packet requires only one MAC layer transmission. When a packet collides or the MAC layer CRC fails (due to an error), that packet must be retransmitted. Therefore, as the number of MAC layer transmissions per packet increases the available bandwidth decreases.

- Packet Loss due to Buffer Overflow: When the medium is busy, packets are queued. If the packet generation rate is greater than the throughput of the medium, the packet buffer overflows and packets must be dropped.

- Packet Loss due to MAC layer failures: This is the number of packets that fail to send after seven MAC layer retries.

- Erroneous Bits: This is the percentage of bits in error within a voice packet. The decoded voice quality for a single packet is highly related to the number of bits in error. ${ }^{4}$

In addition to the above metrics, jitter is also important. Jitter is the variance in inter-arrival time between packet reception at the destination. In general, jitter can be traded off for

\footnotetext{
${ }^{4}$ The decoded voice quality is highly dependent on the decoder's ability to conceal errors. It also depends on exactly which bits are in error. The most important voice data bits should be protected by the MAC layer CRC, as described in Section IV-C
} 


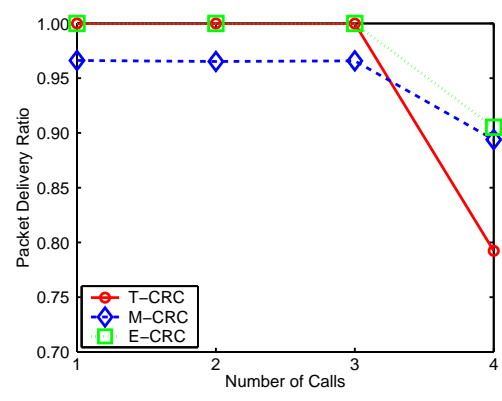

(a) Packet Delivery Ratio

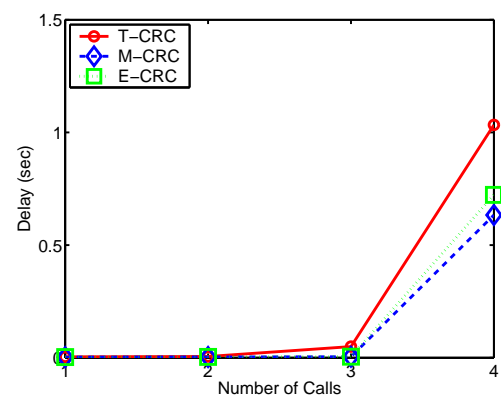

(b) Delay

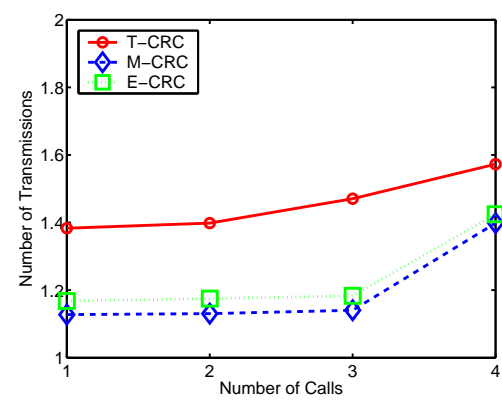

(c) MAC Layer Transmissions per Packet Delivered

Fig. 5. Default scenario.

TABLE IV

Call Quality, Loss, and Delay Requirements.

\begin{tabular}{|c|c|c|}
\hline Quality & Loss $^{5}$ & Delay \\
\hline \hline Toll & $1 \%$ & $80 \mathrm{~ms}$ \\
\hline Business & $3 \%$ & $180 \mathrm{~ms}$ \\
\hline Low & $10 \%$ & $400 \mathrm{~ms}$ \\
\hline
\end{tabular}

additional delay. This is done by buffering packets before playback at the destination.

To isolate the effect of these metrics, we define three call qualities: toll, business and low. In [24] the requirements for toll and business quality calls are detailed. Toll quality has the highest requirements for loss and delay, followed by business and low quality. In [14] the authors describe low quality requirements. The loss and delay requirements for each call quality are summarized in Table IV. In addition, to the voice requirements shown, the ITU has published other requirements and methods to measure voice quality [25], [26].

\section{B. General Results}

The packet delivery ratio for the default scenario is shown in Figure 5(a). The packet delivery ratio is $100 \%$ for E-CRC and $\mathrm{T}-\mathrm{CRC}$ for up to and including three calls. The packet delivery ratio for M-CRC is lower, around $97 \%$. These packet losses are a result of errors in the IP and UDP header, as described in Section IV-B. At four calls all MAC layer strategies encounter higher losses. The E-CRC and M-CRC strategies have nearly $10 \%$ packet loss, meeting the low quality loss requirement, while T-CRC clearly has the lowest packet delivery ratio.

Surprisingly, the reason for the packet losses was not due to seven unsuccessful transmission attempts at the MAC layer. For all experiments in this paper, this number was very

\footnotetext{
${ }^{5}$ The acceptable loss percentage is highly dependent on the voice encoding and the voice decoder's ability to hide errors and losses. For example, [1] provides an optimized G.711 voice decoder capable of acceptable reconstructed speech with up to $30 \%$ packet loss.
}

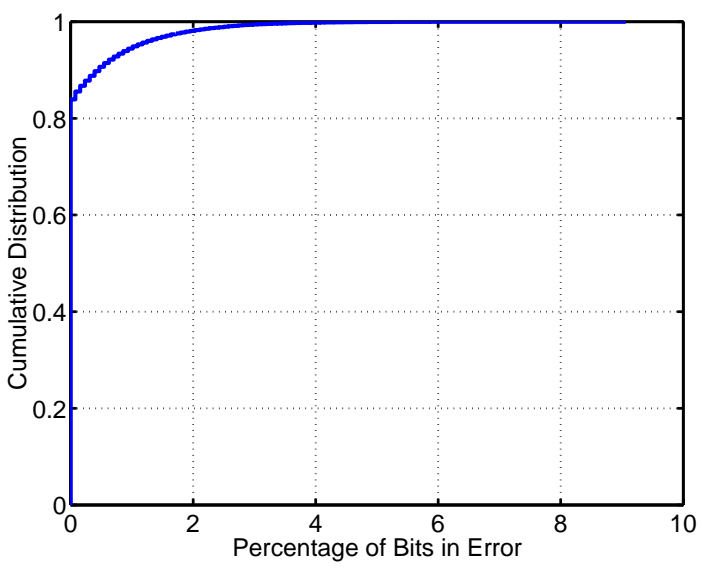

Fig. 6. Cumulative distribution function of packet bit errors.

small. This indicates that the error model did not cripple the IEEE 802.11 MAC layer and that it was successful at delivering packets in less than seven transmissions. At high call rates, the packet losses were a result of buffer overflow, because the maximum capacity of the channel was reached. T-CRC has more than double the packet loss of M-CRC and E-CRC at four calls. This occurs because the IEEE 802.11 MAC protocol cannot support the required bandwidth; many unsuccessful MAC layer transmissions occur when T-CRC is employed.

Focusing on the mean packet delay, shown in Figure 5(b), all MAC CRC strategies perform acceptably up to three calls. The packet delay is less than $50 \mathrm{~ms}$ and the jitter is smaller than the $20 \mathrm{~ms}$ packet generation interval. At four calls the delay experienced is too large to fulfill any of the acceptable call quality requirements because the channel is over-utilized.

Looking at mean number of MAC layer transmissions per packet, shown in Figure 5(c), we see that M-CRC has the fewest transmissions. E-CRC follows M-CRC closely, while T-CRC has a much higher number of transmissions per packet delivered. In terms of the total number of transmissions 


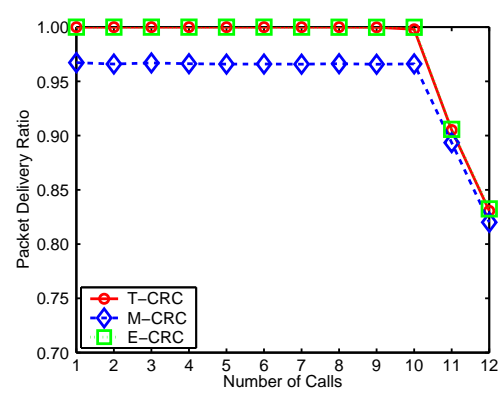

(a) Packet Delivery Ratio

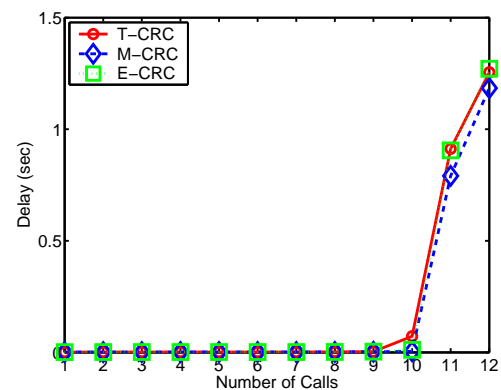

(b) Delay

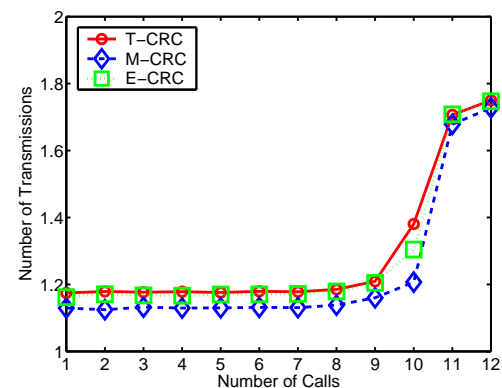

(c) MAC Layer Transmissions per Packet Delivered

Fig. 7. G.723.1 encoder results.

between the three strategies, T-CRC requires nearly $25 \%$ more transmissions than M-CRC, while E-CRC requires only 3\% more. M-CRC has the fewest transmissions because errors are allowed in all parts of the packet except the MAC header. ECRC performs similar to M-CRC because this CRC strategy protects only the MAC, IP and UDP headers. Therefore, differences between the two are caused by errors in the IP and UDP header, which results in a MAC layer retransmission if E$\mathrm{CRC}$ is used. T-CRC has the largest number of retransmissions because an error anywhere in the packet (MAC header, IP header, UDP header or data) causes a retransmission to occur.

Figure 6 shows the cumulative distribution function of the percentage of voice bits per packet that are erroneous for ECRC in the three call scenario. We see that over $80 \%$ of the packets were received without any errors. In the packets that do contain bit errors, only a small percentage of the bits are erroneous. Even the packet with the most errors had fewer than $10 \%$ of its bits in error. Error concealment techniques should prevent this small amount of error from significantly impacting voice quality. The number of bits in error in delivered packets for the E-CRC and M-CRC strategies are comparable, since they both allow bit errors in the data portion. The T-CRC strategy prevents bits errors in the received packets.

\section{Voice Encoder}

There are many characteristics of the voice traffic that are dependent on the encoder. For example, G.723.1 has a much lower bit rate than G.711. With a lower bit rate per call a higher number of calls can be supported. In Figure 7 the packet delivery ratio, mean delay and mean number of transmissions are shown when the G.723.1 encoder is employed. When compared to G.711 (Figure 5), it is easy to see that G.723.1 allows a much larger number of calls to be supported; up to ten calls can be supported with little or no change in performance. At more than ten calls the number of retransmissions per data packet becomes extremely high. This is caused by a combination of factors, including the fact that the MAC layer throughput saturates as the maximum bandwidth is reached.
At low call rates the trends for MAC layer strategies utilizing G.723.1 are similar to that of G.711. At high call rates the advantages of utilizing different MAC layer CRC strategies are proportionately smaller. With G.711 encoding, packets contain 160 bytes of voice data, while with G.723.1 encoding each packet contains only 24 bytes of data. Both G.711 and G.723.1 have the same MAC, IP and UDP headers. Therefore, when utilizing G.723.1 a larger percentage of each packet is the header. Because errors in the MAC headers are not allowed in any MAC layer CRC strategy, the performance impact of allowing errors is smaller with G.723.1 than when G.711 is utilized. This is because a larger portion of each packet is the header. Therefore, when employing G.723.1, the E-CRC and M-CRC strategies show less performance improvement over T-CRC.

\section{Voice Frame Size}

The framing of voice packets effects the packet size. An increase in the amount of voice in each packet increases the packet size and decreases the number of packets per second, when the bit rate is held constant. To examine the effect of varying packet size we simulated multiple packet frame sizes for the G.711 encoder, as shown in Table III. Figure 8 shows the effect of frame size for packet delivery ratio, delay and number of transmissions. In these simulations the number of calls was set to three; at this call rate the wireless channel is stressed but not saturated.

As the frame size increases the packet delivery ratio drops for T-CRC, and the number of transmissions rises steeply. The decease in performance when T-CRC is used is due to the larger packet size. Larger packets are more likely to contain errors. Since T-CRC does not allow any errors, it requires more transmissions. Compared to T-CRC, the effectiveness of M-CRC and E-CRC increases. In terms of the number of transmissions, at $40 \mathrm{~ms}$ voice frames, T-CRC transmits as much as 1.5 times as many frames as M-CRC. As packet size increases, the performance advantage of using a modified MAC CRC strategy becomes more significant. 


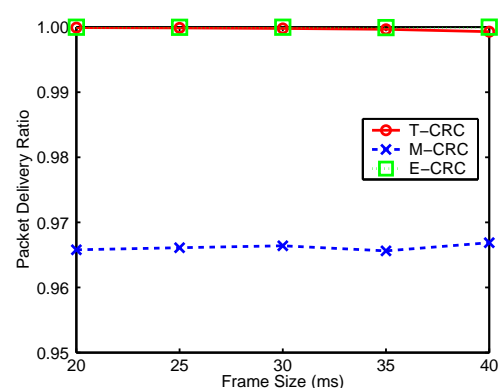

(a) Packet Delivery Ratio

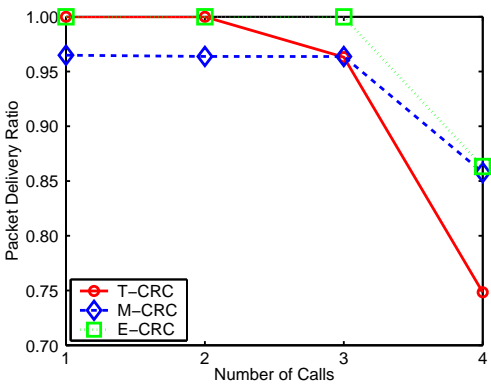

(a) Packet Delivery Ratio

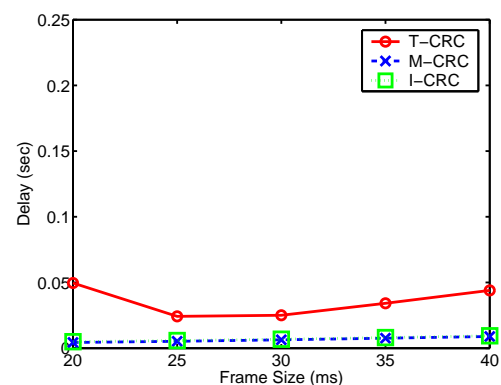

(b) Delay

Fig. 8. Voice frame size results.

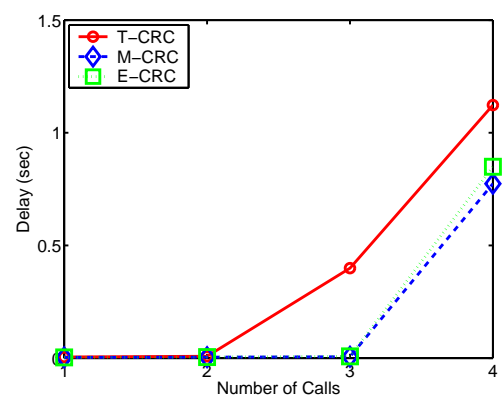

(b) Delay

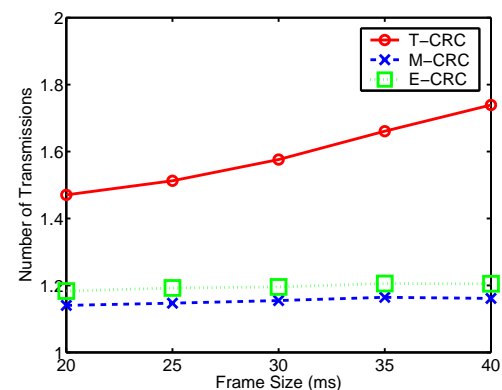

(c) MAC Layer Transmissions per Packet Delivered

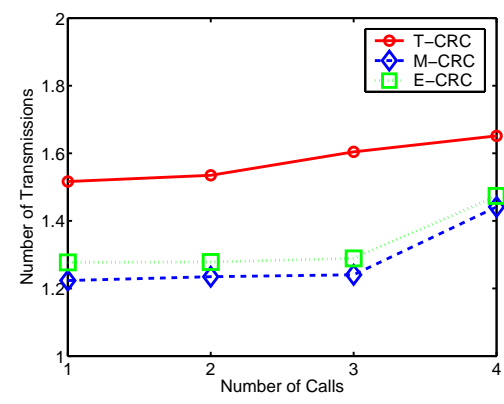

(c) MAC Layer Transmissions per Packet Delivered

Fig. 9. Interference Limited (IL) error model results.

Figure 8(b) shows the delay of M-CRC and E-CRC is low compared to T-CRC. When T-CRC is utilized, the delay increases as the frame size increases. This is due to congestion at the MAC layer. In addition to large frame sizes, for T-CRC a small frame size actually increases the overhead to the point of causing congestion, since a large portion of each packet is the header. For this reason T-CRC at a frame size of $20 \mathrm{~ms}$ actually performs worse than when larger frame sizes are utilized.

\section{E. Error Model Parameters}

When the four sets of error model parameters shown in Table II were simulated, FSFL had the fewest errors and showed little difference between the MAC layer CRC strategies. Since there were few errors, there was little advantage to allowing bit errors. Between the Average and NL error models, there was no significant difference in results because the average BER is the same. When utilizing the IL error model parameters (the error model with the highest BER), the effect of the proposed MAC layer CRC strategies was more significant. Figure 9 shows the results when the IL error model parameters were utilized. Comparing these to the Average case shown in Figure 5, T-CRC has a lower packet delivery ratio, higher delay and higher number of

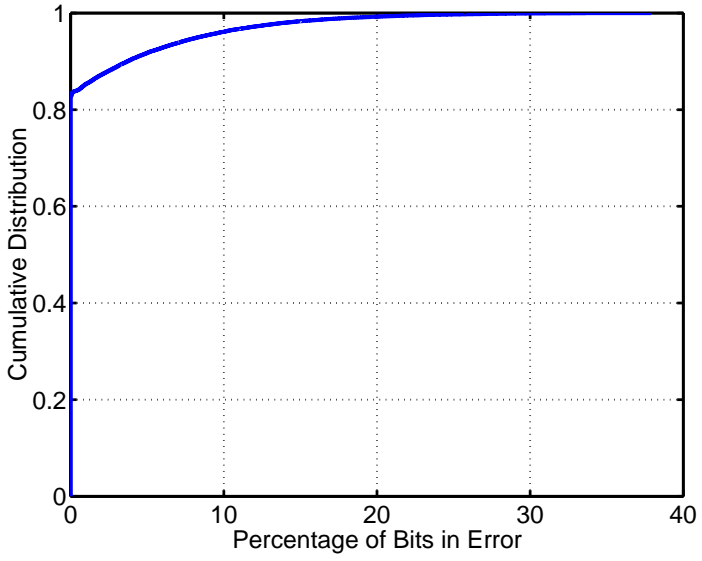

Fig. 10. Cumulative distribution function of packet bit errors when using the Interference Limited (IL) error model parameters.

transmissions. The performance of M-CRC and E-CRC was not significantly impacted. T-CRC performance was decreased for all performance measures at three and four calls. When the channel is more prone to errors, the performance advantage of employing a modified MAC CRC strategy is more evident. 
Figure 10 shows the cumulative distribution function of the percentage of voice bits per packet that are erroneous for ECRC in the three call scenario when using the IL error model. The number of errors in packets when the E-CRC and M-CRC strategies are used is very similar, since they both allow errors in the data portion of packets. We see that over $80 \%$ of the packets are received without any errors. In the packets that do contain bit errors, most have only a small percentage of bits that are erroneous. Comparing Figures 6 and 10, the number of bit errors in the received packets is much larger with the IL error model because this model introduces more errors. A larger number of bits in error will result in worse voice quality, but by properly using error protection (to protect the most important bits) and error concealment techniques, this should not significantly impact the user experience.

\section{CONCLusions ANd Future Work}

In this paper we proposed modifications to the MAC layer that allow bit errors in voice packets, analyzed the effect of these changes under a variety of conditions, and showed that allowing bit errors decreases packet losses, delay and the number of packet transmissions. This results in better call quality and, in some cases, the ability to support a larger number of calls, especially in highly erroneous wireless conditions.

In the future, we plan to evaluate simulated bit errors and the reconstructed speech samples for various encoders. We also plan to continue examination of the effect of allowing bit errors in multi-hop IEEE 802.11 networks [27]. Additionally, we will examine advanced encoding and decoding techniques to conceal bit errors. Furthermore, VoIP is just one application that benefits from allowing bit errors. Audio and video streaming, as well as other multimedia applications, can realize similar improvement in WLANs through the allowance and concealment of errors.

\section{ACKNOWLEDGMENT}

This work is supported in part by a UC Core \Intel Corporation grant and by NSF Infrastructure grant EIA - 0080134.

\section{REFERENCES}

[1] "Global IP Sound," http://www.globalipsound.com/.

[2] International Telecommunication Union, "ITU-T Recommendation G.723.1: Speech coders : Dual rate speech coder for multimedia communications transmitting at 5.3 and $6.3 \mathrm{kbit} / \mathrm{s}, " 1996$.

[3] Markus Kaindl and Norbert Görtz, "AMR Voice Transmission Over Mobile Internet," in Proceedings of the International Conference on Acoustics, Speech and Signal Processing (ICASSP), Orlando, Florida, May 2002, pp. 2049-2052.

[4] B. W. Wah, X. Su, and D. Lin, "A Survey of Error Concealment Schemes for Real-time Audio and Video Transmissions Over the Internet," in Proceedings of the International Symposium on Multimedia Software Engineering, Taipei, Taiwan, December 2000, pp. 17-24.
[5] IEEE Computer Society, "IEEE 802.11g Standard, IEEE Standard For Information Technology," 2003.

[6] P. Garg, R. Doshi, R. Greene, M. Baker, M. Malek, and M. Cheng, "Achieving Higher Throughput and QoS in 802.11 Wireless LANs," in Proceedings of the International Performance Computing and Communications Conference (IPCCC), Phoenix, Arizona, April 2003.

[7] A. A. Abouzeid, S. Roy, and M. Azizoglu, "Comprehensive Performance Analysis of a TCP Session Over a Wireless Fading Link with Queuing," IEEE Transactions on Wireless Communications, March 2003.

[8] H. M. Chaskar, T. V. Lakshman, and U. Madhow, "TCP Over Wireless With Link Level Error Control: Analysis and Design Methodology," IEEE/ACM Transactions on Networking, vol. 7, no. 5, pp. 605-615, 1999.

[9] S. Bhandarkar, N. Sadry, A. L. N. Reddy, and N. Vaidya, "TCP-DCR: A Novel Protocol for Tolerating Wireless," Texas A\&M University Computer Engineering Group, Tech. Rep. TAMU-ECE-2003-01, 2003.

[10] C. Hoene, I. Carreras, and A. Wolisz, "Voice Over IP: Improving the Quality Over Wireless LAN by Adopting a Booster Mechanism - An Experimental Approach," in Proceedings of SPIE 2001 - Voice Over IP (VoIP) Technology, Denver, Colorado, USA, August 2001, pp. 157-168.

[11] L.-A. Larzon, M. Degermark, and S. Pink, "UDP Lite for Real Time Applications," HP Laboratories Bristol, Tech. Rep. HPL-IRI-1999-001, April 1999.

[12] M. Handly and H. Schulzrinne and E. Schooler and J. Rosenberg, "SIP: Session Initiation Protocol," RFC 2543, March 1999.

[13] International Telecommunication Union, "ITU-T Recommendation H.323: Packet-based Multimedia Communications Systems," 2000.

[14] P. C. Mehta and S. Udani, "Overview of Voice over IP," University of Pennsylvania, Tech. Rep. MS-CIS-01-31, February 2001.

[15] International Telecommunication Union, "ITU-T Recommendation G.711: Pulse Code Modulation (PCM) of Voice Frequencies," 1988.

[16] IEEE Computer Society, "IEEE 802.11 Standard, IEEE Standard For Information Technology," 1999.

[17] F. A. Tobagi and L. Kleinrock, "Packet Switching in Radio Channels: Part-II - The Hidden Terminal Problem in Carrier Sense Multiple Access Models and the BusyTone Solution," IEEE Transactions on Communications, vol. 23, no. 12, pp. 1417-1433, December 1975.

[18] G. Bianchi, "Performance Analysis of the IEEE 802.11 Distributed Coordination Function," IEEE Journal Selected Areas in Communications, vol. 18, March 2000.

[19] E. N. Gilbert, "Capacity of a Burst-noise Channel," Bell Systems Technical Journal, vol. 39, pp. 1253-1265, September 1960.

[20] M. Zorzi, R. R. Rao, and L. B. Milstein, "On the Accuracy of a Firstorder Markov Model for Data Transmission on Fading Channels," in Proceedings of ICUPC, Tokyo, Japan, November 1995, pp. 211-215.

[21] E. O. Elliot, "Estimates of Error Rates for Codes on Burst-noise Channels," Bell Systems Technical Journal, vol. 42, pp. 1977-1997, September 1963.

[22] J. J. Lemmon, "Wireless Link Statistical Bit Error Model," Institute for Telecommunication Sciences, Tech. Rep. 02-394, June 2002.

[23] K. Fall and K. Varadhan, "ns Manual," http://wwwisi.edu/nsnam/ns/doc/, 1999, the VINT Project.

[24] Avaya, "Avaya Voice Quality Network Requirements," Avaya Inc., Tech. Rep., August 2002.

[25] International Telecommunication Union, "P.800 Methods for subjective determination of transmission quality," 1996.

[26] ITU, "P.862 Perceptual evaluation of speech quality (PESQ), an objective method for end-to-end speech quality assessment of narrowband telephone networks and speech codecs," 2001.

[27] H. Dong, I. D. Chakeres, A. Gersho, E. M. Belding-Royer, and J. D. Gibson, "Selective Bit-error Checking at the MAC layer for Voice Over Mobile Ad hoc Networks with IEEE 802.11," in Proceedings of the IEEE Wireless Communications and Networking Conference (WCNC), Atlanta, GA, March 2004. 